\title{
Efficacy and Tolerability of the Nasal Airway Stent in the Treatment of Snoring
}

\author{
Takao Miyoshi, MSc ${ }^{1,2}$, Ikumi Sasaki, MT ${ }^{1}$, Fumika Koike, MSc ${ }^{1}$, Saki Oyama, MT $^{1}$, Wakaba \\ Furuie, $M T^{1}$, Miho Akaza, MD, PhD', Tetsuo Sasano, MD, PhD', Meiyo Tamaoka, MD, \\ PhD', Yasunari Miyazaki, MD, PhD', Naohiko Inase, MD, PhD' and Yuki Sumi, MD, PhD ${ }^{1^{*}}$
}

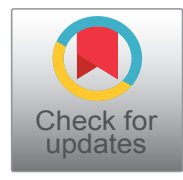

${ }^{1}$ Respiratory and Nervous System Science, Graduate School of Medical and Dental Sciences, Tokyo Medical and Dental University, Japan

${ }^{2}$ Department of Respiratory Medicine, Tokyo Medical and Dental University, Japan

*Corresponding author: Yuki Sumi, MD, PhD, Respiratory and Nervous System Science, Graduate School of Medical and Dental Sciences, Tokyo Medical and Dental University, 1-5-45 Yushima, Bunkyo-ku, Tokyo 113-8519, Japan, Tel: +81-35803-5372, Fax: +81-3-5803-0165

\begin{abstract}
Introduction: The nastent ${ }^{\mathrm{TM}}$ classic, a nasal airway stent (NAS), is a tube-shaped medical device that is positioned from the nose to the soft palate near the uvula. It is expected to reduce snoring by preventing the narrowing and obstruction of the airway and reduce apnea/hypopnea index $(\mathrm{AHI})$ in patients with obstructive sleep apnea (OSA).

Objective: We aimed to examine the tolerability and effectiveness of NAS in snoring.

Methods: We prospectively enrolled subjects who had not been treated for sleep apnea syndrome (SAS) and had no serious complications. We examined the effectiveness of NAS by recording the snoring sounds. A questionnaire survey on the pain caused by NAS treatment was conducted.

Results: Out of 23 subjects recruited, 18 completed the study. Most of the subjects showed improvements of snoring measured as the percentage of snoring time in their total sleep time, average snoring volume, maximum snoring volume, and snoring index (SI, percentage of snoring time in total sleep time multiplied by average snoring volume) after the use of NAS. In time, the subjects got accustomed to wearing the NAS, presenting fewer complaints of discomfort and improvement in sleep in most cases. Out of 23 subjects recruited, five subjects showed an $\mathrm{AHI}>5$. However, only two subjects who showed an $\mathrm{AHI}>5$ completed the study.

Conclusion: The NAS helped to reduce the snoring loudness and duration in most subjects. Pains caused by NAS treatment gradually decreased as the subjects became accustomed to it.
\end{abstract}

\section{Keywords}

Nasal airway stent, Apnea/hypopnea index, Obstructive sleep apnea, Sleep apnea syndrome, Snoring

\section{Abbreviations \\ AHI: Apnea/Hypopnea Index; OSA: Obstructive Sleep Apnea; SAS: Sleep Apnea Syndrome; CPAP: Continuous Positive Airway Pressure; SI: Snoring Index; DI: Discomfort Index}

\section{Introduction}

Obstructive sleep Apnea (OSA) is a sleep-related breathing disorder characterized by recurrent episodes of apnea and/or hypopnea, which leads to decreased oxygen saturation [1]. Snoring occurs because of a partially obstructed airway, typically due to the collapse and vibration of the pharyngeal tissues [2]; whereas, OSA occurs when there is no airflow despite a respiratory effort. Snoring and OSA can have adverse effects even on those other than the patients, such as impaired relationships with spouses or partners [3]. Several lines of evidence have shown that breathing disorders like snoring and obstructive apneas in sleep may be risk factors for ischemic strokes; the natural history of snoring and OSA shows a higher incidence of strokes as compared to undisturbed sleep [4]. Observational clinical studies have shown that the use of continuous

Citation: Miyoshi T, Sasaki I, Koike F, Oyama S, Furuie W, et al. (2019) Efficacy and Tolerability of the Nasal Airway Stent in the Treatment of Snoring. Clin Med Rev Case Rep 6:261. doi.org/10.23937/23783656/1410261

Accepted: March 28, 2019: Published: March 30, 2019

Copyright: (c) 2019 Miyoshi T, et al. This is an open-access article distributed under the terms of the Creative Commons Attribution License, which permits unrestricted use, distribution, and reproduction in any medium, provided the original author and source are credited. 
positive airway pressure (CPAP) is associated with lower rates of cardiovascular complications and death from cardiovascular causes, especially among patients adhering to the treatment $[5,6]$. On the other hand, there is a report that therapy with usual care and CPAP, as compared to usual care alone, did not prevent cardiovascular events in patients with moderate-tosevere OSA and established cardiovascular disease [7].

Not all patients with OSA can be covered by public health insurance in Japan. A considerable proportion of patients may not able to tolerate the CPAP therapy in the long term because of its side effects, discomfort, or inconvenience [8-11]. The use of oral appliances (OA) is also a treatment modality for OSA. OA is an effective treatment in some patients with mild to moderate OSA and is associated with fewer side effects and greater patient satisfaction than CPAP [12]; however, CPAP reduces the $A H I$ more than $O A$ can. There are many dropouts among the patients using $\mathrm{OA}$ as well as those using CPAP $[12,13]$.

The nastent ${ }^{\mathrm{TM}}$ classic [Seven dreamers laboratories, Tokyo, Japan https://nastent.sevendreamers.com/en/], a nasal airway stent (NAS), is a tube-shaped medical device that is inserted through the nose (Figure 1a). The tip of the tube reaches the soft palate near the uvula. The inserted tube aids breathing by preventing obstruction of the airway, frequent arousal, and sleeplessness [14]. NAS is constructed of resilient silicone rubber in order to relieve the symptoms. NAS is expected to be effective in people suffering from simple snoring, in those with OSA and intolerable towards CPAP therapy, as well as OSA patients without public insurance application. In the previous pilot study, NAS proved effective in treating OSA, as determined by the AHI, oxygen desaturation, and snoring in six patients [14]. The tolerability and effectiveness in subjects snoring without OSA have not been reported.

The aim of this study is to assess the tolerability of the NAS and its therapeutic effects on snoring and AHI.

\section{Materials and Methods}

\section{Study subjects}

We prospectively enrolled healthy subjects aged 21-68 years, who agreed to participate in this study. Informed written consent was obtained from all subjects. Subjects were excluded from the study if they had been under SAS treatment. Those who had any kind of severe respiratory, liver, kidney, heart disease, or other serious complications, were also excluded. Subjects who were unable to insert NAS through the nasal cavity for any reasons were also excluded.

\section{Protocol}

Once the subjects had provided written informed consent, they were trained to use the NAS and insert it themselves through their nose (https://nastent. sevendreamers.com/en/about/howto/). The distal end of the NAS is inserted via the nostril into the nasal cavity until it reaches the retropalatal oropharynx usually 10 $\mathrm{mm}$ above the uvula. We observed at subject's throat

(a)

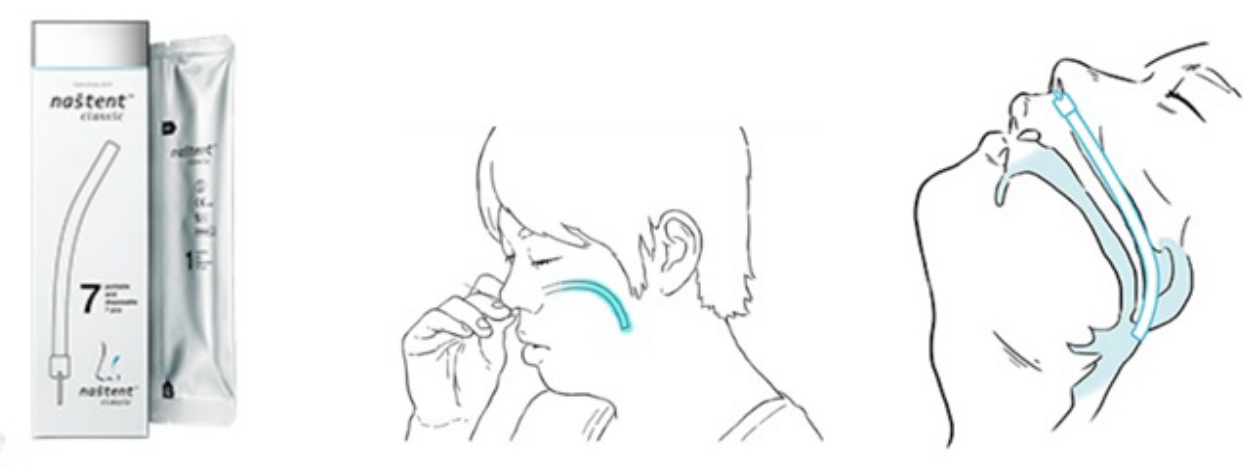

(b)

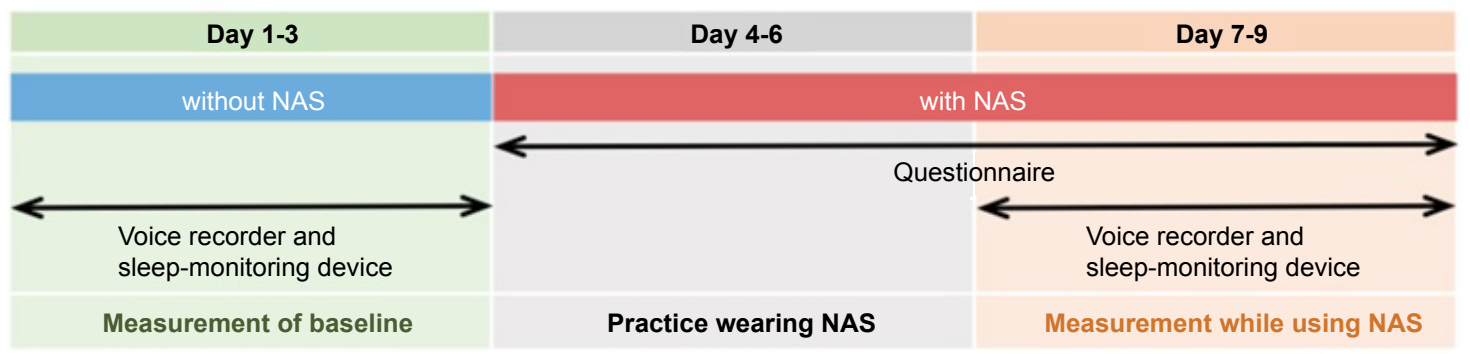

Figure 1: a) Diagrams of the nasal airway stent (NAS). The device is inserted by the users themselves from the nostril until the pharynx is reached, by hooking their nasal septum; b) Overview of the study protocol. 
1. Was it difficult to insert NAS? (not at all/ a little difficult/ difficult/ impossible to insert)

2. Could you fully insert NAS? (yes/no)

3. How long did it to fall asleep? (same as usual/ a little longer than usual/much longer than usual/ impossible sleep)

4. How many times did you wake up at night? (0/ 1/ 2-4/ more than $4 /$ impossible to sleep)

5. How was the quality of sleep using the NAS? (extremely satisfied / a little dissatisfied/ moderately dissatisfied/ extremely dissatisfied)

6. Did you feel sleepy during the day? (not at all/ a little/ vey much/ could not resist drowsiness and took a nap)

7. Select the number for the following question ( $0 \ldots$ no difficulty at all/ $1 \ldots$ little suffering/ $2 \ldots$ high suffering/ $3 \ldots$ intolerable)

- Did you have any pain when inserting NAS?

- Did you have any nausea when inserting NAS?

- Did you have any pain after NAS attachment?

- Did you have any nausea after NAS attachment?

- Did you have any pain when removing NAS?

- Did you have any nausea when removing NAS?

- Did you feel discomfort as NAS hit the wall of the pharynx?

- Did you feel discomfort when swallowing a spit?

Figure 2: Questionnaire survey about the acceptability of nasal airway stent (NAS). Subjects were asked to answer questions from the fourth to ninth day of this study. Discomfort index was the total score of the eight questions in the seventh questionnaire.

at the training of NAS insertion and chose NAS of the appropriate length. The study was conducted for nine days for each subject (Figure 1b). In the initial three days, subjects used a voice recorder and portable sleep-monitoring device (without NAS) to estimate the baseline snoring and $\mathrm{AHI}$. In the next three days, subjects inserted NAS through their nose themselves to get skilled and accustomed during the night, without measurements. In the last 3 days, the effects of NAS usage on snoring were estimated with a voice recorder and the AHI was estimated with a portable sleepmonitoring device. The subjects were asked to fill in a questionnaire assessing the usage of NAS for all days except the first three (Figure 2).

\section{Measurements}

Voice recorder: The sleeping sound was recorded using the digital voice recorder (ICD-PX440, SONY, Tokyo, Japan) placed $50 \mathrm{~cm}$ away from the head. We listened to the recorded sounds directly with our ears and evaluated using the WavePad Audio Editing Software (NCH Software, CO, USA). The loudness and duration of snoring was estimated using Sound Engine (Coderium, Sapporo, Japan). For the loudness, we used arbitrary linear unit which were transformed from $\mathrm{dB}$ displayed on Sound Engine software. We examined parameters such as the percentage of snoring time in total sleep time, average snoring volume, maximum snoring volume, and snoring index (SI: percentage of snoring time in total sleep time multiplied by average snoring volume). Snoring index is a novel measure which can express the snoring volume and duration by a single index.

Sleep-monitoring device: All subjects wore a 4-channel portable sleep-monitoring device (Morpheus $^{\circledR}$, Teijin, Tokyo, Japan, for airflow by cannula, oxygen desat- uration, respiratory movement by respiratory band) to estimate the AHI. The AHI was analyzed automatically with Morpheus Respiratory Software (Teijin), which is established in routine clinical practice.

Tolerability of NAS: We used the questionnaire survey to examine the acceptability of NAS by the subjects (Figure 2). Subjects were asked about difficulty in insertion of NAS and quality of sleeping. The discomfort index (DI) was calculated as the sum of scores with respect to eight questions: pain when inserting NAS, nausea when inserting NAS, pain while fitting NAS, nausea while fitting NAS, pain when removing NAS, nausea when removing NAS, sensation of NAS touching the pharynx, and discomfort in swallowing. The subjects were asked to score the NAS in the assessment questionnaires while using NAS daily [0: no difficulty at all, 1: little suffering, 2: high suffering, 3: intolerable]. The minimum score is 0 and the maximum score is 24 for discomfort index (DI).

\section{Statistical analysis}

Comparisons of data between with NAS and without NAS were analyzed using the paired t-test with GraphPad Prism version 5.0 (http://www.graphpad. com/scientific-software/prism/). Wilcoxon matched pairs test was used for the comparison of DI. P values less than 0.05 were regarded as statistically significant.

This study was approved by the ethics committee of the Tokyo Medical and Dental University (approval number M2015-529).

\section{Results}

\section{Effectiveness of NAS}

Twenty-three subjects including 13 males and 10 females were recruited. The subjects' characteristics 
Table 1: Summary of the subjects in this study.

\begin{tabular}{|c|c|c|c|c|c|c|c|c|}
\hline No. & Gender & Age (year) & Height (cm) & Weight (kg) & Study protocol & AHI & Snoring & NAS size $(\mathrm{mm})$ \\
\hline 1 & Female & 37 & 156 & 50 & completed & 2 & + & 125 \\
\hline 2 & Female & 21 & 155 & 44 & completed & 1 & - & 120 \\
\hline 3 & Male & 21 & 173 & 56 & completed & 3 & - & 130 \\
\hline 4 & Female & 21 & 161 & 47 & completed & 0 & - & 125 \\
\hline 5 & Female & 21 & 155 & 49 & completed & 3 & + & 125 \\
\hline 6 & Male & 53 & 168 & 53 & completed & 2 & + & 140 \\
\hline 7 & Male & 26 & 163 & 80 & completed & 3 & - & 140 \\
\hline 8 & Female & 49 & 157 & 43 & completed & 3 & - & 120 \\
\hline 9 & Male & 25 & 186 & 69 & completed & 3 & + & 140 \\
\hline 10 & Male & 27 & 175 & 95 & completed & 15 & + & 140 \\
\hline 11 & Female & 28 & 153 & 42 & completed & 0 & + & 120 \\
\hline 12 & Female & 63 & 155 & 48 & completed & 0 & + & 120,125 \\
\hline 13 & Male & 25 & 175 & 80 & completed & 4 & + & 130,135 \\
\hline 14 & Female & 36 & 155 & 42 & dropped out & 1 & + & 120 \\
\hline 15 & Male & 68 & 171 & 71 & completed & 1 & + & 135 \\
\hline 16 & Female & 33 & 153 & 44 & dropped out & 1 & - & 120 \\
\hline 17 & Male & 30 & 173 & 76 & completed & 0 & + & 130 \\
\hline 18 & Male & 43 & 178 & 67 & completed & 2 & - & 135 \\
\hline 19 & Male & 24 & 162 & 52 & completed & 8 & + & 130 \\
\hline 20 & Male & 65 & 176 & 67 & completed & 4 & + & 140 \\
\hline 21 & Male & 58 & 174 & 76 & dropped out & 6 & & 140 \\
\hline 22 & Female & 56 & 167 & 53 & dropped out & 13.5 & & 120 \\
\hline 23 & Male & 62 & 168 & 75 & dropped out & 25 & & 140 \\
\hline
\end{tabular}

AHI: apnea/hypopnea index; NAS: nasal airway stent. In the snoring column, the symbol of + indicate the presence of snoring and - indicate absence of snoring. AHI and snoring were recorded without NAS. Subject Nos. 22 and 23 refused to participate in this study at the beginning; hence, their $\mathrm{AHI}$ was referred from results of a previous examination.

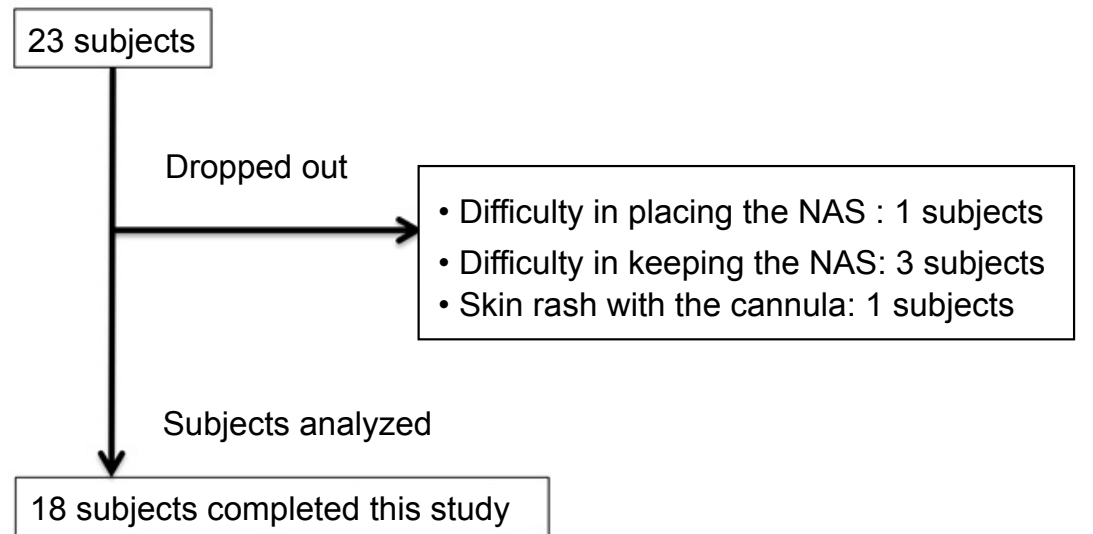

Figure 3: Flow-chart of subjects recruitment.

are summarized in Table 1 . One subject had difficulty in placing or keeping the NAS in the nose (Subject No.21), while one had a skin rash due to contact with the cannula of the portable sleep-monitoring device (Subject No.22); hence, they dropped out of this clinical study at the beginning. Furthermore, three subjects complained of intolerable pains while using NAS (Subject No.14,16,23), thus, they gave up during the study. Finally, 18 subjects were able to use the NAS and complete the study (Figure 3).
Among them, the effectiveness of NAS in the reduction of snoring could be evaluated in 11 subjects because they snored in their sleep. Five subjects showed an $\mathrm{AHI}$ score $>5$, out of which three subjects gave up during the study. Regarding the two subjects analyzed, one revealed an increased $\mathrm{AHI}$ and one showed decreased $\mathrm{AHI}$ after the use of NAS.

The percentages of snoring time in total sleep time were reduced significantly by using the NAS in three 
(a)

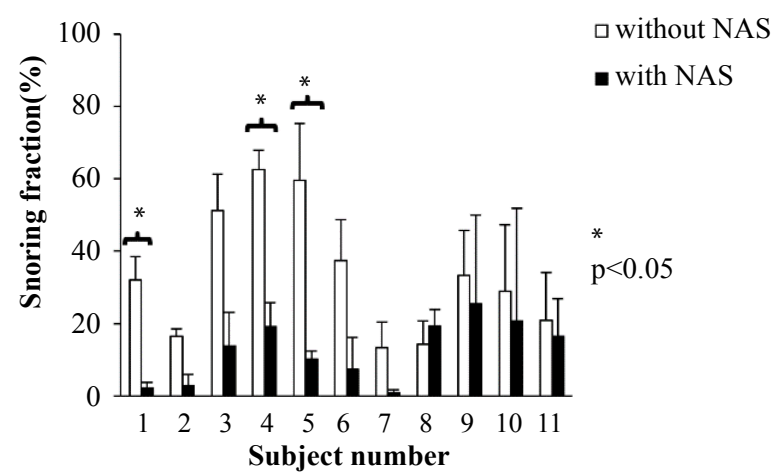

(c)

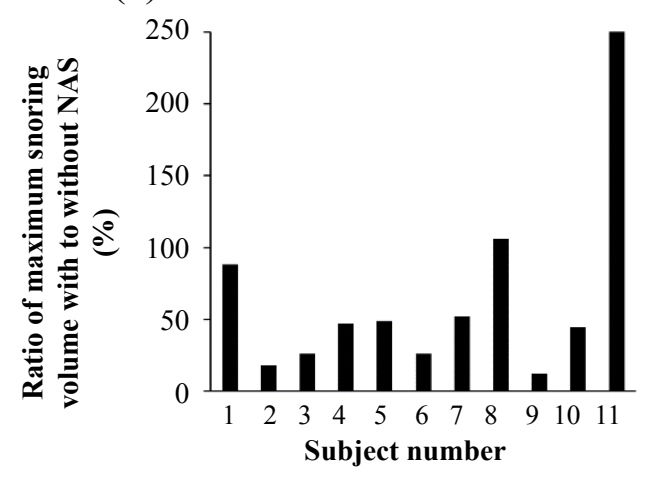

(b) Arbitrary Unit

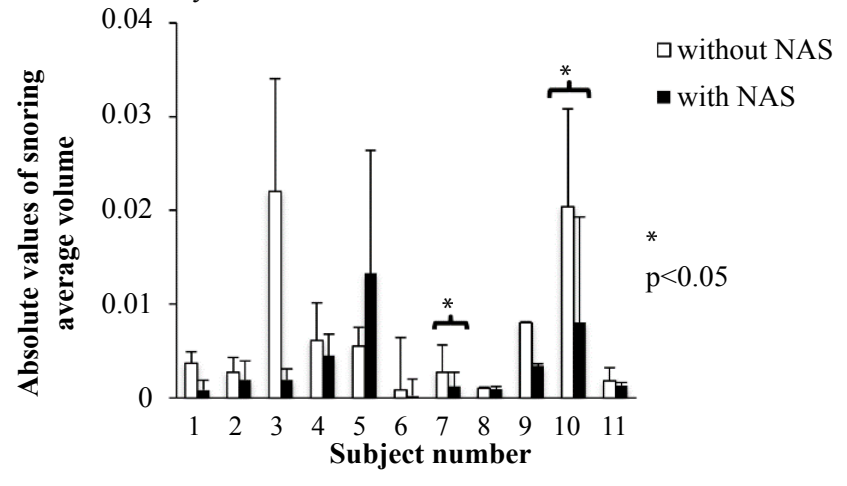

(d)

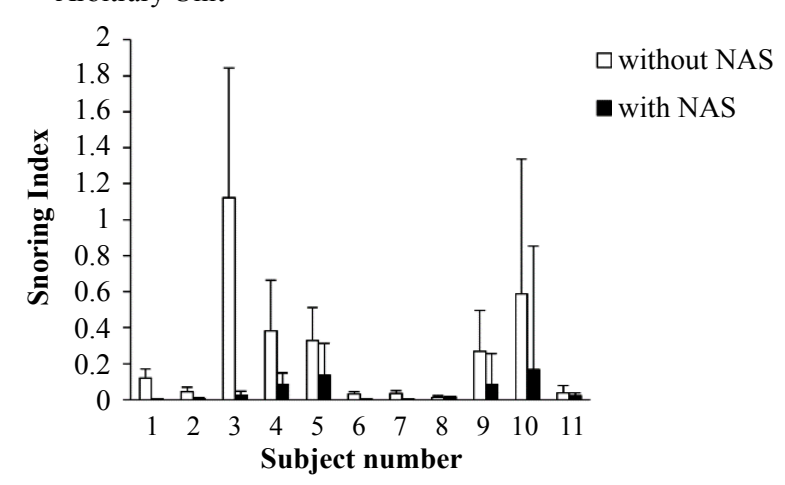

Figure 4: a) The percentages of snoring time in total sleep time with and without nasal airway stent; b) The absolute values of average snoring volume with and without nasal airway stent; $c$ ) The ratio of maximum snoring volume with to without nasal airway stent; d) Snoring index: Percentage of snoring time in total sleep time multiplied by average snoring volume.

subjects (27\%) (Figure 4a). Seven subjects (64\%) showed a reduction in the percentage of snoring time with the use of NAS, but the difference was not significant. There was an increase in the percentage snoring time only in one subject. The average volume of snoring were reduced significantly by using the NAS in two subjects (18\%) (Figure 4b). Eight subjects (73\%) showed a reduction in the average volume of snoring with the use of NAS, but the difference was not significant. The ratio of maximum snoring volume with NAS to that without NAS was evaluated (Figure 4c). In $82 \%$ of the subjects, the maximum snoring loudness had reduced.

In this study, we defined the fraction of snoring time multiplied by average loudness as SI. The average change rate of SI before and after the use of NAS was $29.9 \pm 35.5 \%$, which was the significant decrease $(p=$ 0.02) (Figure $4 d$ ).

\section{Tolerability of NAS}

The results of the questionnaire regarding difficulty in insertion of NAS is shown in Figure $5 \mathrm{a}$. On the first day of insertion (Day 4), $71 \%$ of the subjects complained of difficulty in inserting the NAS. However, on the next day, $66 \%$ of the subjects reported no difficulty in inserting NAS at all. The time taken to fall asleep is shown in Figure $5 b$. On the first day (Day 4), $81 \%$ of the subjects took longer time to fall asleep than usual, but as the days passed, this number reduced and finally $67 \%$ of the subjects were able to fall asleep as usual after five days (Day 9). The number of wake-ups during the night is shown in Figure $5 \mathrm{c}$. As the days passed, the number of subjects who had not woken up in the night increased. On the last day, every subject woke up once or zero during the night. The time course of the discomfort index (DI) was shown in Figure $5 \mathrm{~d}$. The DI on the sixth day (Day 9) had significantly reduced as compared to the first day (Day 4) $(p<0.001)$. Three subjects, who could not complete the study, showed high levels of the $\mathrm{DI}$, although they showed lower DI on the second day (Day 5) as compared to the first day (Day 4). The DI was not significantly different between subjects snoring and those not snoring.

\section{Discussion}

The percentages of snoring time in total sleep time reduced in $91 \%$ of the subjects $(27 \%$ of the subjects showed a significant reduction) by inserting NAS through the nasal cavity. Additionally, the average snoring volume and maximum snoring volume also decreased in most of subjects. We defined $\mathrm{SI}$ as the multiplying percentage of snoring time by the average snoring volume, which would reflect the real world snoring. Almost all subjects showed decreased SI except one subject, who had very low SI from the beginning. Thus, although he showed increased SI after the use of NAS, both snoring time and remained low level almost 
(a)

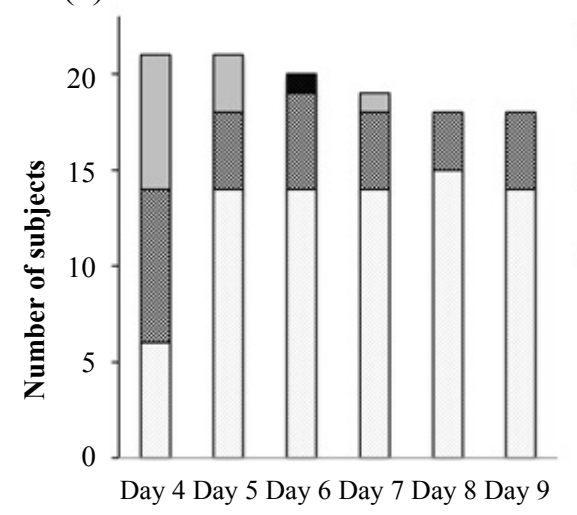

(c)

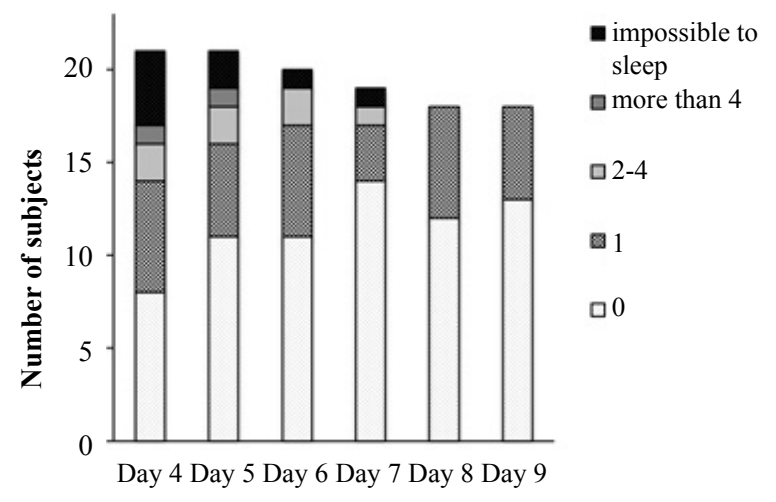

(b)

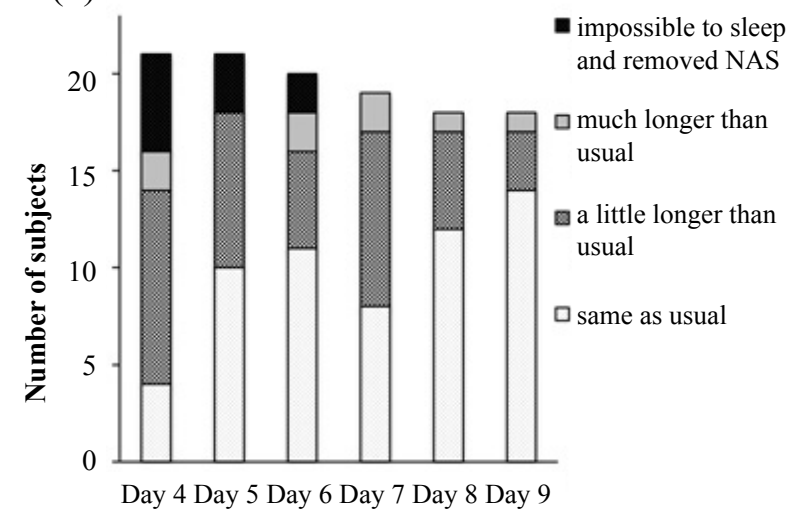

(d)

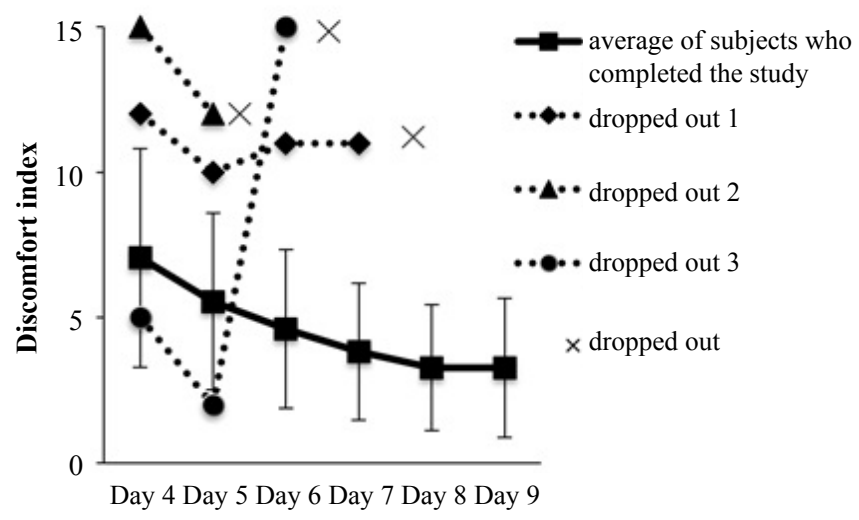

Figure 5: The questionnaire results for six days of nasal airway stent (NAS) use: a) The difficulty in inserting NAS; b) The time taken to fall asleep; c) The number of wake-ups during the night; d) The time course of the discomfort index.

negligible.

Previous study looked at the effect of NAS on patients with OSA [14]. In this study, we looked at tolerability and snoring reduction in general population, therefore, participants were not preselected on snoring/OSA status and as a result, most of subjects did not show high $\mathrm{AHI}$. There were only two subjects with $\mathrm{AHI}>5$ who could complete this study. Therefore, we could not perform statistical analysis and estimate the reduction of AHI. However, previous pilot study showed NAS was effective in reducing AHI [14].

Overall $78 \%$ of subjects had used the NAS for six days. Two subjects complained of considerable discomfort and refused this study at the beginning; three subjects, who complained of considerable discomfort from the first day of NAS use, gave up after the third day. The subjects, who were able to continue up to the last day, got accustomed to it and felt less discomfort as the days passed.

A significant proportion among patients with OSA cannot tolerate CPAP, and NAS could be an alternative treatment for them. Those causing disturbance to others by snoring are advisable to try the NAS for a week to reduce snoring.

In the questionnaire, two other problems of using NAS were reported: The length of the tube and clogged nasal mucus in the tube. It was reported that clogged nasal mucus made nasal breathing difficult and caused discomfort. Furthermore, the sounds of nasal mucus moving through the tube were recorded to be almost at the same level as snoring in some subjects.

The limitations of our study is that the number of participants was small and study population were predominantly without OSA. The total sleep time was determined by the total recording time on the voice recorder and this might be not actual sleeping time determined by EEG.

In future research, increasing the number of subjects with and without OSA, extending the research period while using NAS, and recording EEG would be needed.

\section{Conclusion}

In conclusion, the NAS can reduce the loudness and duration of the snoring in most of the subjects. The NAS was a tolerable device among a large portion of the subjects, and they gradually got used to the fitting of the NAS.

\section{Acknowledgement}

We would like to thank Editage (www.editage.jp) for English language editing.

\section{Authorship Statement}

Authors' contributions Ikumi Sasaki and Yuki Sumi 
designed the study and contributed to collection of data. Takao Miyoshi and Yuki Sumi contributed to collect, analysis and interpretation of data, and wrote the manuscript. All other authors have contributed to collect and interpret the data. All authors approved the final version of the manuscript and agree to be accountable for all aspects of the work in ensuring that questions related to the accuracy or integrity of any part of the work are appropriately investigated and resolved.

\section{Disclosure Statement}

The authors declare no conflicts of interest associated with this manuscript.

\section{References}

1. Gaspar LS, Álvaro AR, Moita J, Cavadas C (2017) Obstructive sleep apnea and hallmarks of aging. Trends Mol Med 23: 675-692.

2. Al-Hussaini A, Berry S (2015) An evidence-based approach to the management of snoring in adults. Clin Otolaryngol 40: $79-85$.

3. Cartwright RD, Knight S (1987) Silent partners: The wives of sleep apneic patients. Sleep 10: 244-248.

4. Clarenbach P, Wessendorf T (2001) Sleep and stroke. Rev Neurol (Paris) 157: S46-S52.

5. Campos-Rodriguez F, Martinez-Garcia MA, de la CruzMoron I, Almeida-Gonzalez C, Catalan-Serra P, et al. (2012) Cardiovascular mortality in women with obstructive sleep apnea with or without continuous positive airway pressure treatment: a cohort study. Ann Intern Med 156: 115-122.

6. Marin JM, Carrizo SJ, Vicente E, Agusti AG (2005) Longterm cardiovascular outcomes in men with obstructive sleep apnoea-hypopnoea with or without treatment with continuous positive airway pressure: An observational study. Lancet 365: 1046-1053.

7. McEvoy RD, Antic NA, Heeley E, Luo Y, Ou Q, et al. (2016) CPAP for Prevention of Cardiovascular Events in Obstructive Sleep. N Engl J Med 375: 919-931.

8. Kohler M, Smith D, Tippett V, Stradling JR (2010) Predictors of long-term compliance with continuous positive airway pressure. Thorax 65: 829-832.

9. Truby H, Edwards BA, O'Driscoll DM, Young A, Ghazi L, et al. (2018) Sleeping Well Trial: Increasing the effectiveness of treatment with continuous positive airway pressure using a weight management program in overweight adults with obstructive sleep apnoea-A stepped wedge randomised trial protocol. Nutr Diet 76: 110-117.

10. Campos-Rodriguez F, Martinez-Alonso M, Sanchez-de-laTorre M, Barbe F, Spanish Sleep Network (2016) Long-term adherence to continuous positive airway pressure therapy in non-sleepy sleep apnea patients. Sleep Med 17: 1-6.

11. Chai CL, Pathinathan A, Smith B (2006) Continuous positive airway pressure delivery interfaces for obstructive sleep apnoea. Cochrane Database Syst Rev 18: CD005308.

12. Ferguson KA, Ono T, Lowe AA, Keenan SP, Fleetham JA (1996) A randomized crossover study of an oral appliance vs nasal-continuous positive airway pressure in the treatment of mild-moderate obstructive sleep apnea. Chest 109: 1269-1275.

13. Giles TL, Lasserson TJ, Smith BH, White J, Wright J, et al. (2006) Continuous positive airways pressure for obstructive sleep apnoea in adults. Cochrane Database Syst Rev 25: CD001106.

14. Yumi Hirata, Makoto Satoh (2015) Pilot study of a nasal airway stent for the treatment on obstructive sleep apnea. $J$ Sleep Disord Ther 4: 1000207. 